UC3M Working Papers

Economics

18-04

June, 2018

ISSN 2340-5031
Departamento de Economía Universidad Carlos III de Madrid

Calle Madrid, 126

28903 Getafe (Spain)

Fax (34) 916249875

\title{
TULLOCK CONTESTS REWARD INFORMATION ADVANTAGES
}

\author{
A. Aiche ${ }^{1}$, E. Einy ${ }^{2}$, O. Haimanko ${ }^{2}$, D. Moreno ${ }^{3}$, A. Selay ${ }^{2}$, and B. Shitovitz ${ }^{1}$
}

\begin{abstract}
In Tullock contests in which the common value of the prize is uncertain, information advantages are rewarded: if a player $i$ has better information about the value than some other player $j$, then the payoff of $i$ is greater or equal to the payoff of $j$, regardless of the information of the other players.
\end{abstract}

Keywords: Tullock Contests, Common Value, Information Advantage.

JEL Classification: C72, D44, D82

1Department of Economics, University of Haifa

2 Department of Economics, Ben-Gurion University of the Negev

3 Departamento de Economía, Universidad Carlos III de Madrid 


\section{Introduction}

In a Tullock contest - see Tullock (1980) - a player's probability of winning the prize is the ratio of the effort he exerts and the total effort all players exert. We show that information advantages are rewarded in Tullock contests in which the players' common value for the prize is uncertain: if a player $i$ has better information than some other player $j$, then the expected payoff of player $i$ is greater than or equal to that of player $j$. This result holds for any two players with rankable information fields, regardless of the information endowments of the other players in the contest. The arguments behind our result rely on the proof of a theorem of Einy, Moreno and Shitovitz (2002) showing that information advantages are rewarded in the Bayesian Cournot equilibria of oligopolistic industries with linear costs, and on the well known formal equivalence between a Tullock contest and a Cournot oligopoly see, e.g., Szidarovszky and Okuguchi (1997).

\section{Tullock Contests}

In a (common-value) Tullock contest a group of players $N=\{1, \ldots, n\}$, with $n \geq 2$, compete for a prize by simultaneously choosing their efforts, $x=\left(x_{1}, \ldots, x_{n}\right) \in \mathbb{R}_{+}^{n}$. The prize is awarded to the players in a probabilistic fashion: if $x \in \mathbb{R}_{+}^{n} \backslash\{0\}$, then the probability that player $i \in N$ wins the prize is $\rho(x)=x_{i} / \sum_{k=1}^{n} x_{k}$, whereas if $x=0$, then the prize is allocated according to some fixed probability vector $\rho(0) \in \triangle^{n}$. Players' uncertainty about the value of the prize is described by a probability space $(\Omega, \mathcal{F}, p)$, where $\Omega$ is the set of states of nature, $\mathcal{F}$ is a $\sigma$-field of subsets of $\Omega$, and $p$ is a probability measure on $(\Omega, \mathcal{F})$ representing the players' common prior belief. Players' common value for the prize is an $\mathcal{F}$-measurable and bounded random variable $V: \Omega \rightarrow \mathbb{R}_{++}$. The private information of player $i \in N$ is described by a $\sigma$-subfield of $\mathcal{F}$, which we denote by $\mathcal{F}_{i}$. This means that for any event $A \in \mathcal{F}_{i}$ player $i$ knows whether the realized state of nature is contained in $A$; in particular, if $\mathcal{F}_{i}$ is generated by a finite or countably infinite partition of $\Omega$, then $i$ knows the element of the partition containing the realized state of nature.

A Tullock contest defines a Bayesian game in which the set of actions of each player is $\mathbb{R}_{+}$, and for $\omega \in \Omega$ and $x \in \mathbb{R}_{+}^{n}$ the payoff of player $i \in N$ is

$$
u_{i}(\omega, x)=\rho(x) V(\omega)-x_{i} .
$$


(Note that if the cost of effort is of the form $W(\omega) x$, where $W$ is a non-negative random variable such that inf $W>0$, then the resulting payoff function is an affine transformation of the function (1). Hence our results also hold in this case, i.e., when the players constant marginal cost of effort $W$ is uncertain.) In this game, a pure strategy for player $i \in N$ is an $\mathcal{F}_{i}$-measurable and integrable function $X_{i}: \Omega \rightarrow \mathbb{R}_{+}$, which describes $i$ 's choice of effort in each state of nature. (The measurability restriction implies that player $i$ can condition his effort only on his private information.) An equilibrium of a Tullock contest is a Bayesian Nash equilibrium of the Bayesian game defined by the contest. We restrict attention to pure strategies.

\section{Reward to Information Advantages}

The proposition below shows that (with some qualification) information advantages are rewarded in Tullock contests. For any $\mathcal{F}$-measurable random variable $f$, we denote by $\mathbb{E}\left[f \mid \mathcal{F}_{i}\right]$ a random variable which is (a version of) the conditional expectation with respect to the $\sigma$-field $\mathcal{F}_{i}$ - see, e.g., Borkar (1995) for a formal definition. Also for any profile of strategies $X=\left(X_{1}, \ldots, X_{n}\right)$, we denote by $X_{-i}$ the profile obtained from $X$ by suppressing the strategy of player $i$.

Proposition. Let $X$ be an equilibrium of a Tullock contest such that inf $\sum_{j=1}^{n} X_{j}>0$. If player $i$ has an information advantage over player $j$, i.e., $\mathcal{F}_{j} \subset \mathcal{F}_{i}$, then

$$
\mathbb{E}\left[u_{i}(\cdot, X(\cdot)] \geq \mathbb{E}\left[u_{j}(\cdot, X(\cdot)]\right.\right.
$$

Proof. For any strategy profile $X=\left(X_{1}, \ldots, X_{n}\right)$ and state of nature $\omega \in \Omega$, the payoff of each player $i \in N$ may be written as

$$
\begin{aligned}
u_{i}(\omega, X(\omega)) & =\frac{X_{i}(\omega)}{\sum_{j=1}^{n} X_{j}(\omega)} V(\omega)-c\left(X_{i}(\omega)\right) \\
& \left.=P\left(\omega, \sum_{j=1}^{n} X_{j}(\omega)\right) X_{i}(\omega)\right)-C\left(\omega, X_{i}(\omega)\right),
\end{aligned}
$$

where the functions $P: \Omega \times \mathbb{R}_{++} \rightarrow \mathbb{R}_{+}$and $C: \Omega \times \mathbb{R}_{+} \rightarrow \mathbb{R}_{+}$are defined as

$$
P(\omega, x)=\frac{V(\omega)}{x},
$$


and $C(\omega, x)=x$. Thus, if $X$ is an equilibrium of the contests, then $X$ is an equilibrium of the symmetric oligopolistic industry in which the players are the firms, the inverse market demand is the function $P$, and the firms' cost is $C$.

Einy, Moreno and Shitovitz (2002) show that information advantages are rewarded in any equilibrium of an oligopolistic industry under certain conditions on the inverse demand and cost functions. Although the function $P$ in $(2)$ does not satisfy these conditions, the proof of Einy, Moreno and Shitovitz (2002) applies to the present setting provided that

$$
\mathbb{E}\left[1_{X_{i}>0} \times \frac{d}{d x_{i}} u_{i}(\cdot, X(\cdot)) \mid \mathcal{F}_{i}\right]=0
$$

holds for every $i \in N$. This equation immediately yields equation (2.6) of Einy, Moreno and Shitovitz (2002), page 157, from which point on the proof applies without change.

We establish equation (3). For any $\varepsilon \in \mathbb{R}$ define $X_{i, \varepsilon}^{\prime}:=\max \left\{X_{i}+\varepsilon, 0\right\}$. Then for every $\omega \in \Omega$

$$
\begin{aligned}
& \lim _{\varepsilon \rightarrow 0+} 1_{X_{i}>0}(\omega) \cdot \frac{u_{i}\left(\omega, X_{-i}(\omega), X_{i, \varepsilon}^{\prime}(\omega)\right)-u_{i}(\omega, X(\omega))}{\varepsilon} \\
= & \lim _{\varepsilon \rightarrow 0+} 1_{X_{i}>0}(\omega) \cdot \frac{u_{i}\left(\omega, X_{-i}(\omega), X_{i,-\varepsilon}^{\prime}(\omega)\right)-u_{i}(\omega, X(\omega))}{-\varepsilon} \\
= & 1_{X_{i}>0}(\omega) \cdot \frac{d}{d x_{i}} u_{i}(\omega, X(\omega)),
\end{aligned}
$$

where for $x \in \mathbb{R}_{+}^{n}$

$$
\frac{d}{d x_{i}} u_{i}(\omega, x)=\frac{\sum_{j \in N \backslash\{i\}} x_{j}}{\left(x_{i}+\sum_{j \in N \backslash\{i\}} x_{j}\right)^{2}} V(\omega)-1 .
$$

Since $V$ has bounded support, there exists $b>\bar{v}:=\sup V$. It follows that $X_{i}$ is bounded from above by $b$ almost everywhere on $\Omega$, as otherwise the expected equilibrium payoff of player $i$ would be negative conditional on some positive-measure event $A_{i} \in \mathcal{F}_{i}$, making it profitable for player $i$ to deviate to $Y_{i}=1_{\Omega \backslash A_{i}} \times X_{i}$. Write inf $\sum_{i \in N} X_{i}:=a>0$. Since for every $\omega$ the function $u_{i}(\omega, x)$ is concave in $x_{i}$, for any $\varepsilon \in\left(0, \frac{a}{2}\right)$ and $\omega \in \Omega$

$$
\left|\frac{u_{i}\left(\omega, X_{-i}(\omega), X_{i, \varepsilon}^{\prime}(\omega)\right)-u_{i}(\omega, X(\omega))}{\varepsilon}\right| \leq \max \left\{\left|\frac{d}{d x_{i}} u_{i}(\omega, X(\omega))\right|,\left|\frac{d u_{i}}{d x_{i}}\left(\omega, X_{-i}(\omega), X_{i, \varepsilon}(\omega)\right)\right|\right\}
$$

and

$$
\left|\frac{u_{i}\left(\omega, X_{-i}(\omega), X_{i,-\varepsilon}^{\prime}(\omega)\right)-u_{i}(\omega, X(\omega))}{-\varepsilon}\right| \leq \max \left\{\left|\frac{d u_{i}(\omega, X(\omega))}{d x_{i}}\right|,\left|\frac{d u_{i}\left(\omega, X_{-i}(\omega), X_{i,-\varepsilon}(\omega)\right)}{d x_{i}}\right|\right\} .
$$


Since $\sum_{j \in N \backslash\{i\}} X_{j}+X_{i,-\varepsilon} \geq \frac{a}{2}$ for $\varepsilon \in\left(0, \frac{a}{2}\right)$, it follows from (5) that the right-hand side functions in both (6) and (7) are bounded from above by $\frac{4 b+2 a}{a^{2}} \bar{v}+1$ when $\varepsilon \in\left(0, \frac{a}{2}\right)$. Using this fact, (4), and the conditional dominated convergence theorem, we obtain

$$
\begin{aligned}
& \lim _{\varepsilon \rightarrow 0+} \mathbb{E}\left[1_{X_{i}>0}(\cdot) \times \frac{u_{i}\left(\cdot, X_{-i}(\cdot), X_{i, \varepsilon}^{\prime}(\cdot)\right)-u_{i}(\cdot, X(\cdot))}{\varepsilon} \mid \mathcal{F}_{i}\right] \\
= & \lim _{\varepsilon \rightarrow 0+} \mathbb{E}\left[1_{X_{i}>0}(\cdot) \times \frac{u_{i}\left(\cdot, X_{-i}(\cdot), X_{i,-\varepsilon}^{\prime}(\cdot)\right)-u_{i}(\cdot, X(\cdot))}{-\varepsilon} \mid \mathcal{F}_{i}\right] \\
= & E\left[1_{X_{i}>0}(\cdot) \times \frac{d}{d x_{i}} u_{i}(\cdot, X(\cdot)) \mid \mathcal{F}_{i}\right] .
\end{aligned}
$$

As $1_{X_{i}>0}$ is $\mathcal{F}_{i}$-measurable and can be extracted from the expectation, by using (6) and (7) with all three terms multiplied by $1_{X_{i}>0}$, and (8), we obtain

$$
\mathbb{E}\left[1_{X_{i}>0}(\cdot) \times \frac{d}{d x_{i}} u_{i}(\cdot, X(\cdot)) \mid \mathcal{F}_{i}\right]=0
$$

The following remark establishes that the qualification in the proposition holds under some general conditions.

Remark. Let $X$ be an equilibrium of a classic Tullock contest in which either $(i) \mathcal{F}_{1}, \ldots, \mathcal{F}_{n}$ are finite, or (ii) $n=2$ and $\inf V>0$. Then $\inf \sum_{j=1}^{n} X_{j}>0$.

Proof. Assume (i) holds. Since $\sum_{i \in N} X_{i}$ is measurable with respect to $\vee_{i \in N} \mathcal{F}_{i}$ (the smallest $\sigma$-field containing each $\left.\mathcal{F}_{i}\right)$, which is finite, the probabilities $p\left(\sum_{i \in N} X_{i} \geq a\right)$ can take only finitely many values in $[0,1]$. Let $\delta=\max _{a>0} p\left(\sum_{i \in N} X_{i} \geq a\right)$, and suppose the maximum is attained at $a_{0}>0$. By Remark $1, \sum_{i \in N} X_{i}>0$ in any equilibrium $X$, and hence $\lim _{a \searrow 0} p\left(\sum_{i \in N} X_{i} \geq a\right)=p\left(\sum_{i \in N} X_{i}>0\right)=1$. Therefore $\delta=1$ and $a_{0}$ is the desired bound for the equilibrium sum of efforts.

Assume (ii) and, w.l.o.g., that player 2 has an information advantage over player 1. Write $\underline{v}:=\inf V$ and $\bar{v}=\sup V$, and let $\varepsilon \in\left(0, \frac{\underline{v}}{12}\right)$. Also, let $a \in(0, \varepsilon)$ be such that

$$
\frac{2 a}{\varepsilon+2 a}<\frac{\varepsilon}{2 \bar{v}}
$$

(Such value exists because the left-hand side vanishes when $a \rightarrow 0+$.) Now consider an equilibrium $X$ of the contest. We will show that $X_{1} \geq a$. Assume by the way of contradiction that $X_{1}<a$. Then there exists a positive-measure set $A_{1} \in \mathcal{F}_{1}$ such that $X_{1}<a$ on $A_{1}$. We show that $X_{2} \leq \varepsilon$ almost everywhere on $A_{1}$. 
Suppose to the contrary that $X_{2}>\varepsilon$ on some positive-measure $A_{2} \in \mathcal{F}_{2}$ which is a subset of $A_{1}$. Consider a strategy

$$
X_{2}^{\prime}=\frac{\varepsilon}{2} \cdot 1_{A_{2}}+X_{i} \cdot 1_{\Omega \backslash A_{2}}
$$

in $S_{2}$. Then, by switching from $X_{2}$ to $X_{2}^{\prime}$ player 2 decreases his expected reward by at most

$\frac{2 a}{\varepsilon+2 a} \bar{v} \cdot p\left(A_{2}\right)$, and simultaneously decreases his expected cost by at least $\frac{\varepsilon}{2} \cdot p\left(A_{2}\right)$. By the choice of $a$, the first expression is smaller than the second, and hence deviating to $X_{2}^{\prime}$ is, in expectation, profitable for player 2 , which contradicts that $X$ is an equilibrium.

It follows that $\max \left\{X_{1}, X_{2}\right\} \leq \varepsilon$ almost everywhere on $A_{1}$. Let $i$ be a player for whom $\mathbb{E}\left[\rho_{i}(X) \mid A_{1}\right] \leq \frac{1}{2}$, and consider a strategy

$$
X_{i}^{\prime \prime}=3 \varepsilon \cdot 1_{A_{1}}+X_{i} \cdot 1_{\Omega \backslash A_{1}}
$$

Since $A_{1} \in \mathcal{F}_{1} \subset \mathcal{F}_{2}, X_{i}^{\prime \prime}$ is measurable with respect to both $\mathcal{F}_{1}$ and $\mathcal{F}_{2}$, and hence with respect to $\mathcal{F}_{i}$. Further, $\rho_{i}(X) \leq \rho_{i}\left(X_{-i}, X_{i}^{\prime \prime}\right)$ almost everywhere on $A_{1}$, and that $E\left(\rho_{i}\left(X_{-i}, X_{i}^{\prime \prime}\right)\right.$ $\left.A_{1}\right) \geq \frac{3}{4}$ (this is due to the fact that, almost everywhere on $A_{1}, \rho_{i}\left(X_{-i}, X_{i}^{\prime \prime}\right) \geq \frac{3 \varepsilon}{3 \varepsilon+\varepsilon}=\frac{3}{4}$ ). Thus, by switching from $X_{i}$ to $X_{i}^{\prime \prime}$ player $i$ increases his expected payoff by at least $\frac{1}{4} \underline{v} \cdot p\left(A_{1}\right)$, while increasing his expected cost by at most $3 \varepsilon \cdot p\left(A_{1}\right)$. By the choice of $\varepsilon$, such a deviation leads to a net gain in the expected payoff, which contradicts that $X$ is an equilibrium. We conclude that, indeed, $X_{1} \geq a$.

\section{References}

[1] Borkar V.S., 1995. Probability theory: an advanced course. Springer.

[2] Einy, E., Moreno, D., Shitovitz, B., 2002. Information advantage in Cournot oligopoly, J. Econ. Theory 106, 151-160.

[3] Szidarovszky, F., Okuguchi, K., 1997. On the existence and uniqueness of pure Nash equilibrium in rent-seeking games. Games Econ. Behav. 18, 135-140.

[4] Tullock, G., 1980. Efficient rent-seeking, in J.M. Buchanan, R.D. Tollison and G. Tullock, Toward a theory of rent-seeking society. College Station: Texas A\&M UP. 